\title{
Population genetic screening programmes: technical, social and ethical issues
}

\author{
On behalf of the European Society of Human Genetics' PPPC
}

European Journal of Human Genetics (2003) 11, 903-905. doi:10.1038/sj.ejhg.5201104

Published online 22 October 2003

\section{Introductory considerations}

Genetic screening is increasingly possible for a large number of disorders. The question of whether or not this approach should be offered at the population level is a challenge to health-care providers, the medical community and policy makers. Genetic screening can be of benefit but can also do harm. The availability of genetic tests at low cost may lead to the systematic offer of screening tests without the appropriate medical environment for providing information prior to testing and counselling afterwards. There is therefore a need to introduce effective and acceptable safeguards, standards and procedures relating to the implementation and organisation of genetic screening programmes. To discuss these issues and produce recommendations from the professional point of view, the Public and Professional Policy Committee (PPPC*) of the European Society of Human Genetics (ESHG) organised a

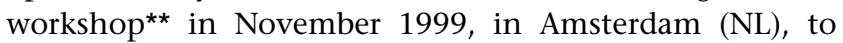
which 51 experts from 15 European countries were invited. They received, prior to the meeting, a working paper developed by the PPPC, which was revised after the meeting to take into account the points of views expressed by the participants. This document is now available on the web site of the ESHG. Following the workshop, the ESHG issued statements and recommendations that were endorsed at the Amsterdam ESHG annual meeting, in May 2000. These recommendations are expected to reflect the views of the scientific community.

\section{Statements and recommendations}

Definition of genetic screening

(1) Genetic screening may be defined as any kind of test performed for the systematic early detection or exclusion of a genetic disease, the genetic predisposition or resistance to a disease, or to determine whether a person carries a gene variant, which may produce disease in offspring.
(2) Screening may be concerned with the general population or with specific subpopulations defined on some basis other than their health.

(3) Screening for genetic conditions or genetic traits predictive of diseases is a medical act. As the public has trust in the professional duty of care, a compliance effect may be expected when a screening test is offered, whose effect underlines the responsibility of professionals offering such tests.

(4) Genetic screening is distinguished from other types of medical screening by the genetic nature of the disorder that may result in risk implications to family members of the person screened, even though family members may not be, nor perhaps wish to be, included in the screening programme. Genetic screening is also distinguished from other forms of screening because its aim is not necessarily to prevent or treat diseases in the person screened; it may be used for health-related reproductive or lifestyle choices.

Potential benefit and harm of genetic screening

(5) The benefits of genetic screening include presymptomatic detection of diseases or susceptibility to diseases for prevention, early diagnosis, care and treatment; the detection of genetic predisposition to adverse effects of environmental factors facilitate avoidance of harm, and detection of carrier status to enable reproductive or lifestyle decision.

(6) The possible detrimental effects of genetic screening include anxiety raised by information that cannot be used to make positive personal choices about therapies or preventive measures, or which is difficult to understand and interpret; undue pressure on individual choice; social stigmatisation of persons at increased genetic risk; social stigmatisation of persons who might decline an offer of genetic screening; disclosure of information about family members who have not consented to testing; misuse of the information and discrimination based on the test results after disclosure to third parties, such as insurers and employers. 


\section{Criteria for introducing genetic screening programmes}

(7) A screening programme should be considered only if there is general agreement on the benefits expected from the programme, both from the point of view of the professionals and from the point of view of the patients and the community; there is an important health problem in terms of number of people affected or severity of the health problem; there is an effective intervention or a decision to be taken by the person screened, dependent on the test results; there is a suitable test with known predictive value.

(8) As there are potential harms attached to genetic screening, a programme should only be considered if benefits clearly outweigh harm. The benefit and harm to the screened person has to be considered first, before considering the relatives, especially when neonatal and childhood screening is concerned. Benefits and harms should have been evaluated by pilot programmes and interpreted taking their cultural dimensions into account.

(9) Before starting any population genetic screening programme, all alternative options have to be explored.

\section{Principles for defining screening programmes}

(10) A genetic screening programme should always be preceded by a pilot phase on a subpopulation with an appropriate evaluation of the positive and negative outcomes at all levels, including evaluation of the test, its acceptability and uptake rate; validity of the test results; use of the test results in decision-making processes; psychosocial consequences of the screening test and its clinical validity; utility of the screening process; staffing necessary to run the programme, total programme cost. The results of the pilot phase have to serve as a basis for a final decision on whether to proceed or not, involving the health-care providers and representatives of the patients in the decision process.

(11) Economic criteria alone cannot be used to justify a screening programme.

(12) Even when benefit outweighs harm, the screening test should be offered as an option and individuals must remain free to refuse the test after appropriate information and counselling.

(13) Newborn screening can be justified and strongly recommended if early diagnosis and treatment clearly benefit the newborn.

(14) The goals and the target population must be well defined. The outcome measures have to be clearly defined and have to be in agreement with the goals. High uptake rate per se is not a sufficient goal.

(15) As the prevalence of genetic traits often varies among populations, screening programmes may be better targeted to subpopulations with high prevalence if the community agrees to have a focused health-care programme. In populations composed of subpopulations with different genetic background, the test should be selected according to population substructure.

(16) Systematic case finding followed by systematic cascade testing is intermediate between population screening and testing of high-risk individuals and should also be considered, according to the same criteria as population genetic screening.

(17) Increasingly, clients are aware of available tests and may ask for them. If these are not part of a validated routine screening program, professionals have to be careful to consider and explain the clinical utility of the test for the individual requesting to be tested.

(18) The principles defined for genetic screening programmes are also relevant for research and pilot programmes.

\section{Organisation of genetic screening programmes}

(19) The promoter of the programme should be a governmental or nongovernmental body that has the authority to ensure that the organisational criteria are met for a defined population, including equity of access, monitoring of practices and the timely provision of appropriate treatments and preventive measures.

(20) Prior to the implementation of the programme, guidelines have to be produced on the techniques to be used, the type of information to be provided, the management structure and the monitoring process. The information leaflets have to be approved by independent bodies in collaboration with lay organisations when they exist.

(21) Any programme should include quality assessment procedures for the laboratory tests, the provision of genetic counselling services and the delivery of information.

(22) Programmes should be monitored to ensure that practices follow agreed guidelines.

(23) Information before and after the test should be provided by trained professionals with appropriate support and should allow the possibility of a face-to-face discussion. Genetic counselling sessions should be provided in case of unfavourable results.

(24) Any programme should offer the possibility of contact with a trained professional for further information (help line).

(25) If treatment or preventive measures exist, they should be offered with a minimum of delay and equitably.

(26) Professionals and the public should have easy access to information on validated screening tests and guidelines.

(27) Rapid advances in technology may make it possible to screen large number of disorders or traits simultaneously. It will then become difficult if not impossible to provide proper information about each of the conditions and traits screened. Our recommendation is to authorise packages only when there is enough consistency in the characteristics of the conditions screened to allow properly informed consent from the consumer. 
(28) It is recommended that an independent coordinating body be established at the International level to address matters of common interest and standards.

This document forms part of a BIOTECH program financed by the Commission of the European Communities (CEE BIO4-CT98-0550).

All rights reserved. No part of this document may be reproduced or utilised in any form or by any means, electronic or mechanical, including photocopying, recording, microcopying, or by any information storage and retrieval system, without permission in writing from the copyright holder.

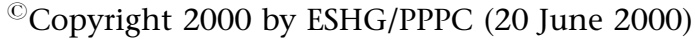

* Members of the PPPC were: Ségolène Aymé (Paris, France), Martin Bobrow (Cambridge, UK), Gerry EversKiebooms (Leuven, Belgium), Peter Farndon (Birmingham,
UK), Helena Kääriäinen (Helsinki, Finland), Ulf Kristoffersson (Lund, Sweden), Marcus Pembrey (London, UK), Sandy Raeburn (Nottingham, UK) Albert Schinzel (Zürich, Switzerland), Joerg Schmidtke (Hannover, Germany), Leo ten Kate (Amsterdam, Netherlands), Lisbeth Tranebjaerg (Copenhagen, Denmark).

** The workshop was organised by Ségolène Aymé (Paris, France), Gerry Evers-Kiebooms (Leuven, Belgium) and Leo ten Kate (Amsterdam, Netherlands).

Correspondence: Professor S Ayme Hôpital Broussais, INSERM SC 11, 102 rue Didot, Paris F-75014, France. Tel: + 3315653 8137; Fax: + 3315653 8138; E-mail:ayme@orpha.net 